\title{
Artificial Intelligence-Based Dynamic Contrast-Enhanced Magnetic Resonance Imaging for Quantitative Evaluation of the Efficacy of Targeted Therapy for Liver Metastasis from Colon Cancer and Nursing
}

\author{
Yanling Zheng $\mathbb{D}$, ${ }^{1}$ Yongqing Cao $\mathbb{D}^{1},{ }^{1}$ Jiheng Liu $\mathbb{D}^{1},{ }^{1}$ Hui Chen $\mathbb{D},{ }^{1}$ and Liming Peng $\mathbb{D}^{2}$ \\ ${ }^{1}$ Department of Hematology and Oncology, Changsha First Hospital, Changsha 410005, China \\ ${ }^{2}$ Department of Nursing, Changsha First Hospital, Changsha 410005, China \\ Correspondence should be addressed to Liming Peng; 1132037@mail.dhu.edu.cn
}

Received 9 July 2021; Revised 12 August 2021; Accepted 14 August 2021; Published 29 August 2021

Academic Editor: Gustavo Ramirez

Copyright () 2021 Yanling Zheng et al. This is an open access article distributed under the Creative Commons Attribution License, which permits unrestricted use, distribution, and reproduction in any medium, provided the original work is properly cited.

\begin{abstract}
The purpose of this study was to quantitatively evaluate the targeted treatment and nursing effect of patients with liver metastasis of colon cancer by enhanced magnetic resonance imaging. First, Dynamic Contrast-Enhanced Magnetic Resonance Imaging (DCE-MRI) images were restored. 35 patients with liver metastases from colon cancer were selected as research subjects and treated by bevacizumab, and high-quality nursing methods were used to improve the psychophysiology of the patients. Enhanced CT examination and DCE-MRI scan were performed before and after the treatment. After the image was processed by the artificial intelligence algorithm, apparent diffusion coefficient (ADC), measurement transmission constant (Ktrans), reflux constant (Kep), extravascular extracellular volume fraction ( $\mathrm{Ve}$ ), and other data on the image were recorded. Two months after the treatment, 35 patients were divided into effective and ineffective groups. It was found that, after treatment, the Kep value and Ktrans value of the effective group were significantly reduced, showing statistically significant differences, and the $P$ value was less than 0.05 , and the Kep value and Ktrans value of the ineffective group showed no significant changes. The Ve value of the two groups gradually decreased with the progress of the treatment. In the effective group, the Ve values before the treatment, on the third day after the treatment, and on the seventh day after treatment were $(0.235 \pm 0.134),(0.338 \pm 0.116)$, and $(0.457 \pm 0.097)$, respectively. Compared with the Ve values of the ineffective group, there was a statistical difference, $F=3.592, P=0.0245$; the ADC value was negatively correlated with the Ktrans and Kep values, and the results were statistically significant. It was concluded that the above indicators are effective in evaluating the efficacy of the targeted therapy of liver metastasis from colon cancer, and DCE-MRI is of great significance in predicting the efficacy of targeted therapy of liver metastasis from colon cancer.
\end{abstract}

\section{Introduction}

Colon cancer is a malignant tumor of the digestive tract. Its incidence ranks third among gastrointestinal tumors after esophageal cancer and gastric cancer. Colon cancer is prone to liver metastasis. It is reported that approximately $15 \%-$ $25 \%$ of patients have liver metastasis when colon cancer is diagnosed [1], and in patients with advanced colon cancer, the probability of liver metastasis is as high as $50 \%-75 \%$. At present, there is not an effective way to treat colon cancer. If detected in the early stage, it can be treated by the surgery.
However, there are not obvious symptoms at the early stage, and it is usually discovered at the middle and late stages where the surgery method alone is no longer effective. By then, antitumor vascular drugs become the main treatment method. Microvessel Density (MVD) and Vascular Endothelial Growth Factor (VEGF) are two parameters widely used in the evaluation of tumor neovascularization [2], which can reflect the growth of tumor and the therapeutic effects after treatment.

Liver metastasis from colon cancer is an obstacle in the treatment, and there has been no universally effective 
treatment plan. Statistics reveal that antitumor drug treatment can significantly improve the survival rate of patients with advanced colon cancer [3]. In addition to targeted drug therapy, chemotherapy and surgery are also required, which challenge the patient's physiology and psychology and aggravate the pain of patients. At this time, the high-quality care is of great significance. It can alleviate the fear and anxiety of patients and reduce the recovery time. How to evaluate the therapeutic effects is a hot spot.

The commonly used methods to evaluate curative effects include KRAS genetic testing, CT, and DCE-MRI. As imaging technology marches forward continuously, DCE-MRI attracts more and more attention. It demonstrates superb capabilities in quantitatively analyzing blood supply. It is a noninvasive imaging method to evaluate tumor neovascularization [4]. However, the DCE-MRI image is always blurry and intervened by noise and artifacts due to limb movement, heartbeat, and breathing. Luckily, image restoration based on partial differential algorithm can improve the quality of the image and enrich image feature information $[5,6]$. The apparent diffusion coefficient (ADC), measured transmission constant (Ktrans), reflux constant (Kep), and extravascular extracellular volume fraction (Ve) on the restored image can be used to evaluate the vascular permeability and perfusion within the tumor [7]. Of them, Kep and Ktrans are closely related to MVD and VEGF [8].

In clinical treatment, the evaluation of the efficacy of targeted therapy for liver metastasis from colon cancer has become a new problem. In this study, patients diagnosed with colon cancer requiring targeted therapy were selected as research subjects for DCE- MRI scans. The algorithm based on partial differential equation can effectively improve the diagnostic effect of other images of MRI on colon cancer. At the same time, it can assist doctors to detect the lesion area in the image. It can not only reduce the workload of manual processing, but also improve the poor diagnostic effect caused by subjective differences, to quantitatively evaluate the therapeutic effects of bevacizumab, expecting to provide theoretical and data support for curative effects evaluation.

\section{Materials and Methods}

2.1. Research Subjects. In the study, from May 2019 to December 2020, 35 patients aged 25-75 years who were diagnosed with liver metastasis from colon cancer were selected as research subjects, including 20 male patients and 15 female patients. All patients had no history of tumor in other parts and had not had chemotherapy. They were diagnosed as having colon cancer through histology.

They were selected as per the following inclusion criteria: (I) patients with no major organ failure; (II) the survival time expected to be over three months; and (III) patients who had signed the informed consent form for targeted therapy.

Exclusion criteria were as follows: (I) those with allergic reactions to targeted drugs; (II) those with a history of mental illness; (III) those without measurable lesions by DCE-MRI scans; and (IV) those with dysfunction of vital organs.

All the research contents in this paper have been approved by the medical ethics committee.
2.2. Targeted Therapy and Inspection Plan. FOLFOX + bevacizumab regimen: oxaliplatin $85 \mathrm{mg} / \mathrm{m}^{2} \mathrm{dl}$; leucovorin $400 \mathrm{mg} / \mathrm{m}^{2} \mathrm{dl}$; intravenous injection of fluorouracil $400 \mathrm{mg} / \mathrm{m}^{2} \mathrm{dl}$; continuous intravenous infusion of fluorouracil $2400 \mathrm{mg} / \mathrm{m}^{2}$ for $46 \mathrm{~h}$; intravenous infusion of bevacizumab $5 \mathrm{mg} / \mathrm{kgdl}$. These operations were repeated every 2 weeks.

The patient had a low-fiber diet three days before the examination and took 2-3 L of the ethylene glycol isotonic solution within 8 hours before the examination. The bowel preparation was completed when the excrement was clear liquid. Otherwise, the enema was performed on the day of the examination. 10 minutes before the examination, $10 \mathrm{~mL}$ of anisodamine was intramuscularly injected. The patient kept lying on the left side for the enema and was slowly infused with $1000-1500 \mathrm{~mL}$ isotonic mannitol solution.

2.3. DCE-MRI Scanning. For each patient participating in the experiment, DCE-MRI scans were performed on the day before targeted therapy, on the third day, and on the seventh day after treatment, respectively. The GE Discovery MR750w HD 3.0 T magnetic resonance was used, with 18channel coil. The gadolinium pentetate meglumine was injected intravenously at $0.25 \mathrm{~mL} / \mathrm{kg}$ in the right elbow, and then $20 \mathrm{~mL}$ of normal saline was used for flushing immediately.

Magnetic resonance pulse sequence scanning was performed before contrast agent injection and dynamic enhanced scanning was performed on coronal or transverse sections after contrast agent injection. The image data obtained after the scan were read by two senior physicians, and finally unified data results were obtained and compared with the pathological staging results. The scope of the tumor was determined through dynamic enhancement scan, and three regions of interest (ROI) were delineated where there were no obvious necrosis and bleeding. The values of Ktrans, Kep, and $\mathrm{Ve}$ were obtained by artificial intelligence algorithm. The scanning time was the $X$-axis, and the signal intensity was the $Y$-axis. The average of the three calculation results was taken as the standard value of quantitative analysis.

After the scan, $10 \mathrm{~mL}$ of fasting blood was drawn from each of 35 patients and processed with sodium citrate. Then, the content of tumor markers in the serum was measured.

\subsection{Differential Equation Algorithm to Restore DCE-MRI} Image. The overall variational image restoration model (TV model) has excellent anisotropic diffusion [9]. It can also perform denoising while restoring the image, and it is relatively simple in calculation.

Assuming that the image to be restored is $F=R \cup E$, the cost function of the image to be restored is expressed as follows:

$$
T(f)=\int_{f} r\left(\left|\nabla_{f}\right|\right) \mathrm{d} x \mathrm{~d} y
$$

where $T(f)$ is the energy universal function obtained from the image $f$ and $\nabla_{f}$ is the existing impact function. 
At the edge of the area that needs to be repaired, there is an impact function $\nabla_{f}$. At this time, the function needs to satisfy the following equation:

$$
\int_{f} r\left(\left|\nabla_{f}\right|\right) \mathrm{d} x \mathrm{~d} y<\infty .
$$

In the differential equation algorithm model, $r\left(\left|\nabla_{f}\right|\right)=\left|\nabla_{f}\right|$. At this time, the function is expressed as follows:

$$
T(f)=\int_{f}\left|\nabla_{f}\right| \mathrm{d} x \mathrm{~d} y .
$$

If denoising is performed in the process of restoring the image, the following conditions must be met:

$$
\delta^{2}=\frac{1}{s(E)_{E}} \int_{E}\left|f-f_{0}\right|^{2} \mathrm{~d} x \mathrm{~d} y
$$

where $\delta^{2}$ is the variance of Gaussian noise; $s(E)$ represents the undamaged part of the image, $f$ is the restored image, and $f_{0}$ is the original image with noise. Combining the equations (3) and (4), the overall energy functional function [10] is obtained.

$$
G_{\lambda}(f)=\int_{f}\left|\nabla_{f}\right| \mathrm{d} x \mathrm{~d} y+\frac{\lambda}{2} \int_{E}\left|f-f_{0}\right|^{2} \mathrm{~d} x \mathrm{~d} y,
$$

where $\lambda$ represents the Lagrange multiplier. Next, the minimum value of the energy functional function of this model is solved.

$$
E(f)=\int_{w} D\left(x, y, f, \frac{\varphi_{f}}{\varphi x}, \frac{\Phi_{f}}{\varphi y}\right) \mathrm{d} x \mathrm{~d} y .
$$

To take the minimum value, the following conditions need to be met:

$$
D_{f}-\frac{\varphi}{\varphi J C} D_{f_{x}}-\frac{\phi}{\varphi y} D_{f_{y}}=0 .
$$

In the TV model in the differential algorithm equation:

$$
D\left(x, y, f, \frac{\varphi_{x}}{\varphi n}, \frac{\varphi_{y}}{\varphi n}\right)=\sqrt{\left(\frac{\varphi_{f}}{\varphi_{x}}\right)^{2}+\left(\frac{\varphi f}{\varphi y}\right)^{2}} \frac{\lambda}{2}\left(f-f_{0}\right)^{2} .
$$

Equation (8) is combined with equation (7) to obtain the minimum energy functional equation of the TV model.

$$
-\nabla \cdot\left(\frac{\nabla_{f}}{\left|\nabla_{f}\right|}\right)+\lambda\left(f-f_{0}\right)=0
$$

For a certain point $z=(x, y) \in \omega$ on the image, the Lagrange multiplier in equation (9) satisfies the following conditions:

$$
\lambda=\left\{\begin{array}{l}
\lambda z \in E \\
O z \in D .
\end{array}\right.
$$

The environment required for training and building the deep learning algorithm model in this research is created by anaconda, Python, and PyCharm software.
2.5. Nursing Management of Liver Metastasis of Colon Cancer. The nursing interventions of rapid rehabilitation surgery in the perioperative period of colon cancer mainly include the following: (1) preoperative: nutritional evaluation, shortening fasting and water time, preoperative psychological intervention, and unconventional mechanical enema; (2) intraoperative: reducing surgical trauma and optimizing anesthesia; (3) postoperative: early in bed activities, early out of bed activities, preventive analgesia, and follow-up after discharge.

2.6. Tumor Staging and Efficacy Evaluation Criteria. Primary tumors are classified into several grades as per international standards [11]. Early tumors that have not spread to other tissues are classified as Tis stage. According to the size and scope of the primary tumor, it is classified as T1-T4. $N$ represents the dissemination of lymph nodes is represented; $N X$ represents that the lymph node status cannot be assessed; N0 represents that there is no lymph node metastasis; $N 1$ represents that there are 1-3 lymph node metastases; and N2 represents that there are 4 or more lymph node metastases.

The curative effect evaluation standard adopts the solid tumor curative effect evaluation standard [12]. If the lesion completely disappears or there is no enhancement for four weeks, it is defined as CR; if the enhancement area is reduced by $30 \%$ and the duration is more than four weeks, it is defined as PR; if the enhancement area does not increase by more than $20 \%$ or the area decreases by less than $30 \%$, it is defined as SD; if the area of one or more lesions increases by more than $20 \%$ or new lesions appear, it is defined as PD. In this study, CR and PR are considered effective, and SD and $\mathrm{PD}$ are considered ineffective.

2.7. Statistics. SPSS 25.0 was used to process the data. The $t$ test was used for statistical analysis of DCE-MRI quantitative parameters, ADC values, and tumor markers of different pathological grades. $P<0.05$ was the threshold for significance.

\section{Results}

3.1. DCE-MRI Examination Results of Targeted Therapy. Of the 35 patients with liver metastases from colon cancer, after two months of targeted therapy (bevacizumab combination therapy), 19 patients were classified into the effective group, and 16 patients were classified into the ineffective group. Figures 1 and 2 are DCE-MRI images of the effective group before and after treatment, and Figures 3 and 4 are the DCE-MRI images of the ineffective group before and after treatment.

The DCE-MRI image of the effective group showed that the number of tumors in the patient was significantly reduced, and the tumor size and area were significantly reduced, while in the ineffective group, 5 patients had worsened conditions after treatment, and their DCE-MRI images showed that the number of tumors increased significantly, and the area of tumors also increased 




(a)

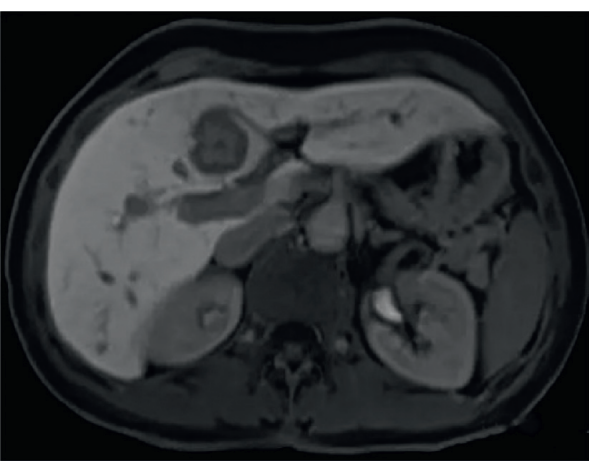

(b)

FIgure 1: The DCE-MRI images in effective group before and after treatment.

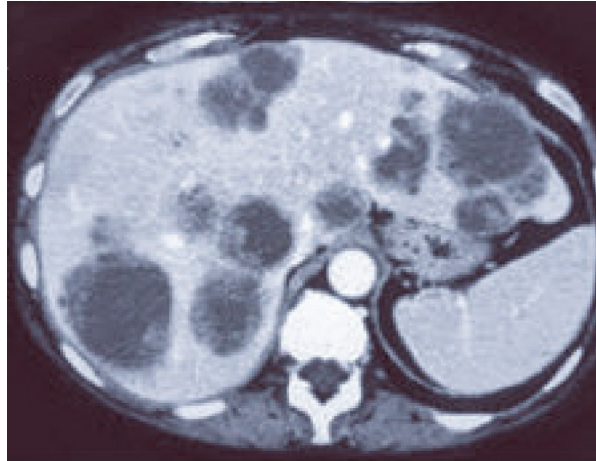

(a)

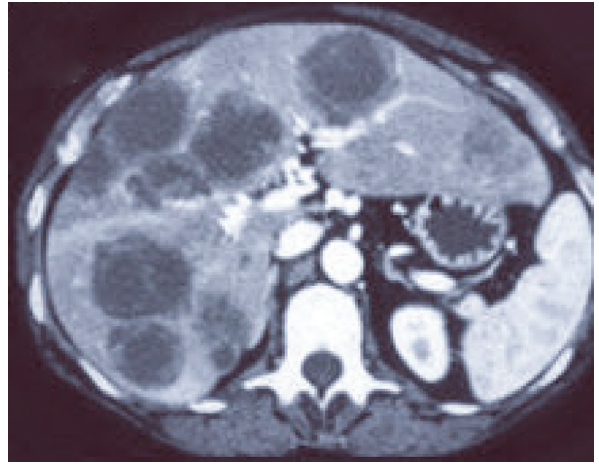

(b)

Figure 2: The DCE-MRI images in ineffective group before and after treatment.

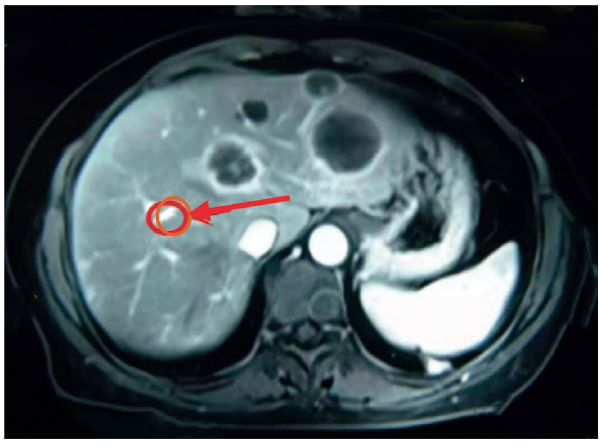

(a)



(b)

FIgure 3: The restoration effects of the damaged DCE-MRI image.

significantly. After treatment, 11 patients were in stable condition, and their DCE-MRI images showed no obvious change in the number of tumors and no significant change in the area of the tumor.

3.2. The Restoration Effect of the Differential Equation Algorithm. A DCE-MRI image of colon cancer that was damaged due to objective reasons was restored, and the restoration effects were analyzed. It was noted from Figure 3 that the differential equation algorithm had good restoration effects on the image, and the restoration time was $47.153 \mathrm{~s}$.

3.3. DCE-MRI Parameters before and after Targeted Therapy. In the effective group, the Ktrans value showed a decrease trend; in the ineffective group, the Ktrans value decreased on the third day after the treatment, and then the value 


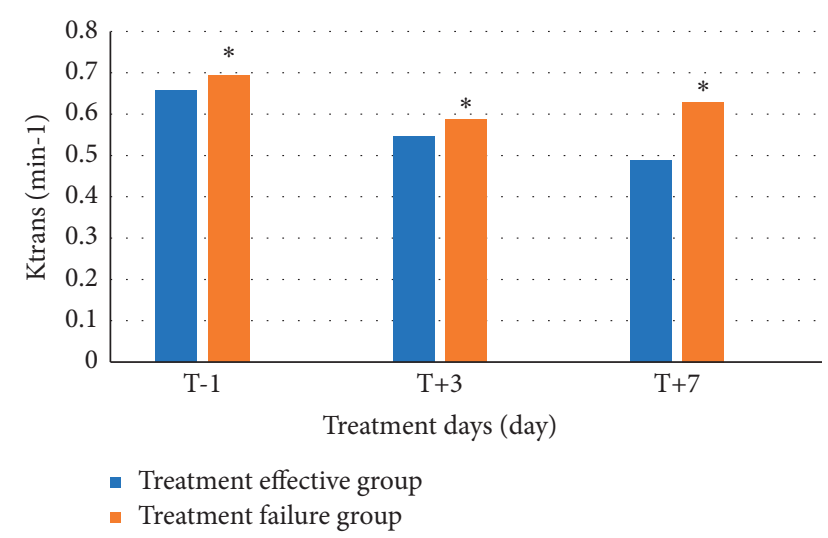

Figure 4: Comparison of Ktrans values before and after treatment in the effective group and ineffective group $\left({ }^{*}\right.$ means that there is a statistically significant difference between the two groups).

gradually recovered. There were statistically significant differences between the two groups (Figure 4).

The Ktrans values of the effective group and the ineffective group were $(0.658 \pm 0.196) \mathrm{min}^{-1}$ and $(0.693 \pm 0.124) \mathrm{min}^{-1}$ detected on the day before treatment, respectively, and there was no statistical significance. On the third day after the treatment, the Ktrans values of the effective group and the ineffective group were $(0.547 \pm 0.126)$ $\min ^{-1}$ and $(0.587 \pm 0.133) \mathrm{min}^{-1}$, respectively. Obviously, Ktrans values of the two groups of patients were significantly reduced, and there were statistically significant differences. On the 7th day after targeted therapy, the Ktrans values of the effective group and the ineffective group were $(0.487 \pm 0.113) \mathrm{min}^{-1}$ and $(0.629 \pm 0.101) \mathrm{min}^{-1}$, respectively. Obviously, the Ktrans value of the ineffective group rose significantly, while the Ktrans value of the effective group still steadily decreased.

As for the Kep value, in the effective group, it rose slightly on the 7th day after the treatment; in the ineffective group, it decreased significantly on the third day after the treatment but then gradually increased. Data analysis revealed that there were statistically significant differences between the two groups of data (Figure 5).

Before treatment, the Kep values in the treatment effective group and the treatment ineffective group were $(0.723 \pm 0.122) \mathrm{min}^{-1}$ and $(0.701 \pm 0.135) \mathrm{min}^{-1}$, respectively, and there was no statistically significant difference between the data. On the third day after targeted therapy, the Kep values of the treatment effective group and the ineffective group were $(0.348 \pm 0.089) \mathrm{min}^{-1}$ and $(0.479 \pm 0.145) \mathrm{min}^{-1}$, respectively. Obviously, the Kep values of the two groups were significantly reduced, and there are statistical differences. On the 7th day after targeted therapy, the Kep values of the effective group and the ineffective group were $(0.354 \pm 0.124) \mathrm{min}^{-1}$ and $(0.657 \pm 0.128) \mathrm{min}^{-1}$, respectively. It was found that the Kep value of the effective group increased slightly, while the Kep value of the ineffective group rose significantly.

As shown in Figure 6, the Ve values of the two groups gradually increased with the progress of the treatment. In the effective group, the Ve values on the day before treatment,

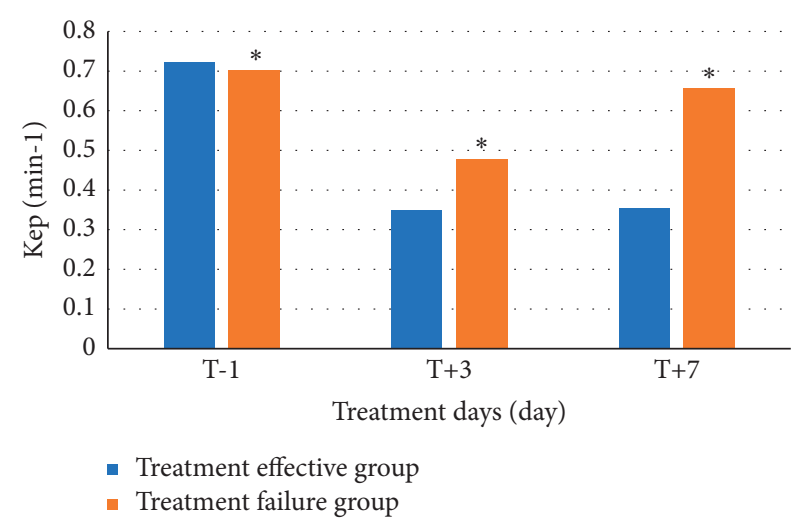

FIGURE 5: Comparison of Kep value of effective group and ineffective group after treatment $(*$ means that there is a statistically significant difference between the two groups).

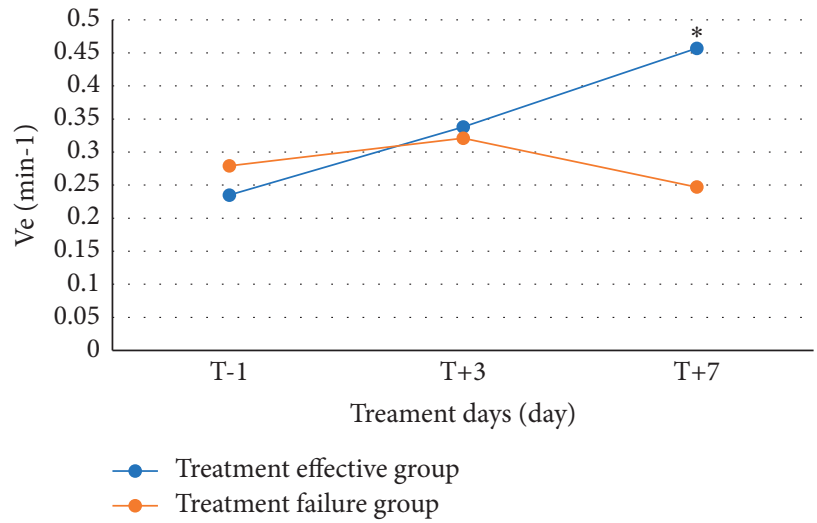

FIGURE 6: Ve values of effective group and ineffective group after treatment $\left({ }^{*}\right.$ means that there is a statistically significant difference between the two groups).

the third day after the treatment, and the seventh day after the treatment were $(0.235 \pm 0.134),(0.338 \pm 0.116)$, and $(0.457 \pm 0.097)$, respectively. Compared with the Ve values of the ineffective group, there was a statistically significant difference.

Before treatment, the ADC values of the effective group and the ineffective group were $(0.00173 \pm 0.00022) \mathrm{min}^{-1}$ and $(0.00170 \pm 0.000135) \mathrm{min}^{-1}$, respectively, and there was no statistically significant difference between the data. On the third day after targeted therapy, the ADC values of the effective group and the ineffective group were $(0.00248 \pm 0.00089) \mathrm{min}^{-1}$ and $(0.00199 \pm 0.000145)$ $\mathrm{min}^{-1}$, respectively. Obviously, the ADC values of the two groups of patients increased significantly, and there were statistically significant differences between the data. On the 7th day after targeted therapy, the ADC values of the effective group and the ineffective group were $(0.00255 \pm 0.000124) \mathrm{min}^{-1}$ and $(0.00178 \pm 0.000128)$ $\min ^{-1}$, respectively.

3.4. Pathology-Related Results before and after Targeted Therapy. Before targeted therapy, the mean MVD counts of 
the effective and ineffective groups were $(10 \pm 0.017)$ bars/ high power lens and (11.323 \pm 0.412$)$ bars/high power lens, respectively. On the $3 \mathrm{rd}$ day after treatment, the mean MVD counts of the two groups were $(7.833 \pm 0.269) /$ high power lens and $(8.189 \pm 0.145) /$ high power lens, respectively; on the 7 th day after treatment, the mean MVD counts of the two groups were $(6.912 \pm 0.177)) /$ high power lens and $(8.937 \pm 0.419) /$ high power lens, respectively (Figure 7$)$.

3.5. The Correlation between the Parameters of DCE-MRI and the Pathological Grading. The pathological grade of the tumor was positively correlated with the Ktrans value, and the results were statistically different $(r=0.783, P<0.05)$; the pathological grade of the tumor was positively correlated with the Kep value, and the results were statistically different $(r=0.794, P<0.05)$; the pathological grade of the tumor was negatively correlated with the Ve value, and the results were statistically different $(r=0.489, P<0.05)$; the pathological grade of the tumor was negatively correlated with the ADC value, and the results were statistically different $(r=-0.647$, $P<0.05)$ (Table 1).

The Ktrans value was positively correlated with the Kep value and MVD count, and the results were statistically different $(r=0.667, P<0.05 ; r=0.713, P<0.05)$.

\section{Discussion}

Colon cancer is a malignant tumor of the digestive tract with a high incidence. It mainly manifests as irregular shadows or lumps on the intestinal wall on medical imaging data. In the early stage, surgical resection has a high success rate in the treatment of colon cancer [13], but there are no obvious symptoms in the early stage, and most patients have developed into the middle and late stage when diagnosed. At this time, surgical removal alone is not effective. Liver metastasis from colon cancer has always been an obstacle in the clinical treatment of colon cancer. To effectively treat liver metastases from colon cancer and improve the survival rate of patients [14], it is a must to remove the metastatic lesions. The primary objective of this study was to quantitatively evaluate the therapeutic effects of targeted therapy in patients with liver metastases from colon cancer. However, in the process of targeted therapy and surgical resection, patients will have various physical and psychological problems. The postoperative infection rate and the patient's mental state are closely related to nursing intervention. High-quality nursing can effectively improve the comprehensive status of patients [15], reduce the occurrence of pneumoperitoneum, and minimize the hospital stay. Therefore, high-quality nursing is required to improve the treatment effects of patients.

Traditional tumor imaging only focuses on the size and morphology of the tumor, and to evaluate the efficacy of targeted therapy requires a new and effective imaging observation method $[16,17]$. DCE-MRI is a new tumor observation method. It uses small molecule contrast agents to observe and analyze tumor blood flow and vascular permeability, and it is possible to determine the impact of

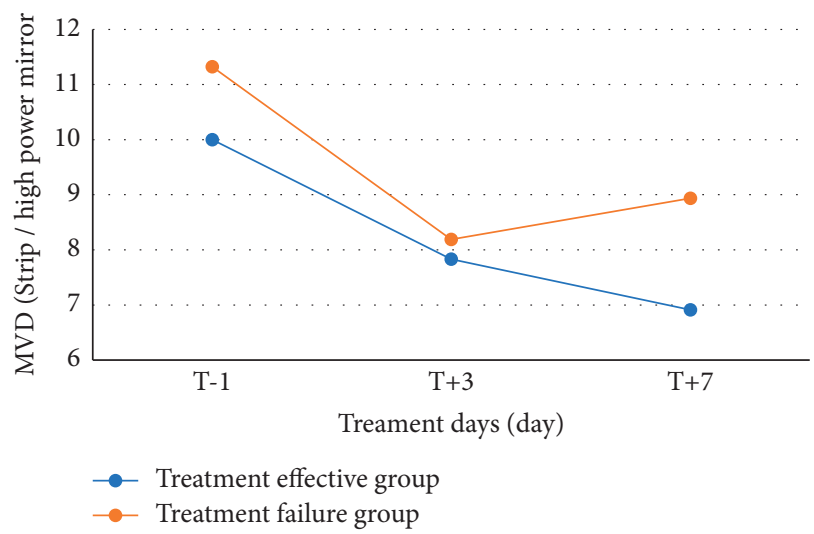

FIgURE 7: MVD counts of the effective group and the ineffective group after treatment.

TABLE 1: Correlation test results.

\begin{tabular}{lcccc}
\hline \multirow{2}{*}{ Pathological grading } & \multicolumn{4}{c}{ DCE-MRI } \\
& Ktrans & Kep & Ve & ADC \\
\hline$r$ & 0.783 & 0.794 & 0.489 & -0.647 \\
$P$ & $\leq 0.001$ & $\leq 0.001$ & 0.036 & 0.002 \\
\hline
\end{tabular}

targeted therapy drugs on tumor blood vessels, so as to judge and analyze the therapeutic effects of targeted therapy. However, in practice, the DCE-MRI image is always intervened by noise and artifacts due to the particularity of the scan site and the different conditions of the patient. Artificial intelligence technology has been widely used in the medical field, and artificial intelligence algorithms can restore medical images $[18,19]$. In this study, the TV model in the differential equation algorithm was applied. However, the image resolution of DCE-MRI technology is not ideal, and generally, the dose of DCE-MRI needs to be reduced as appropriate so as not to affect the tumor vessels [20]. The results of this study showed that the Kep value and Ktrans value of patients in the effective group decreased significantly after treatment, while the Kep value and Ktrans value of patients in the ineffective group did not change significantly after treatment. Therefore, it is speculated that DCEMRI has important significance in predicting and evaluating the efficacy of targeted therapy for colon cancer liver metastasis.

\section{Conclusion}

In this study, the DCE-MRI scan image was restored by the differential equation algorithm, to obtain quantitative parameters of the DCE-MRI scan. Then, the relationship between each parameter and the MVD was analyzed. It was found that the Ktrans and Kep values were positively correlated with MVD, which provided effective data and theoretical support for the subsequent efficacy evaluation of targeted therapy. The ADC value was negatively correlated with the Ktrans and Kep values, and the results were statistically significant. The algorithm based on partial 
differential equation can effectively improve the diagnostic effect of MRI for colon cancer. Dynamic enhanced MRI is of great significance in predicting and evaluating the efficacy of targeted therapy for liver metastasis of colon cancer and can be used to formulate a personalized treatment plan for the clinical treatment.

However, there are still some shortcomings in this study. The sample is too small, which reduces the power of the study. What is more, there are errors caused by human factors. In the follow-up, an expanded sample size is required to strengthen the findings of the study, and the DCEMRI technology should be combined with methods such as ultrasound contrast and other methods to comprehensively evaluate the tumor data.

\section{Data Availability}

The data used to support the findings of this study are available from the corresponding author upon request.

\section{Conflicts of Interest}

The authors declare no conflicts of interest.

\section{Acknowledgments}

This work was supported by Scientific Research Projects of Hunan Health Commission (Project no. 20200216).

\section{References}

[1] M. Riihimäki, A. Hemminki, J. Sundquist, and K. Hemminki, "Patterns of metastasis in colon and rectal cancer," Scientific Reports, vol. 6, no. 1, Article ID 29765, 2016.

[2] J.-H. Pan, S. Zhu, J. Huang et al., "Monitoring the process of endostar-induced tumor vascular normalization by noncontrast intravoxel incoherent motion diffusion-weighted MRI," Frontiers in Oncology, vol. 8, p. 524, 2018.

[3] Y. T. Lee, Y. J. Tan, and C. E. Oon, "Molecular targeted therapy: treating cancer with specificity," European Journal of Pharmacology, vol. 834, pp. 188-196, 2018.

[4] R. A. P. Dijkhoff, R. G. H. Beets-Tan, D. M. J. Lambregts, G. L. Beets, and M. Maas, "Value of DCE-MRI for staging and response evaluation in rectal cancer: a systematic review," European Journal of Radiology, vol. 95, pp. 155-168, 2017.

[5] L. Shi, Y. Zhang, K. Nie et al., "Machine learning for prediction of chemoradiation therapy response in rectal cancer using pre-treatment and mid-radiation multi-parametric MRI," Magnetic Resonance Imaging, vol. 61, pp. 33-40, 2019.

[6] M.-R. Mohammadian-Behbahani and A.-R. Kamali-Asl, "Artificial neural networks approach to pharmacokinetic model selection in DCE-MRI studies," Physica Medica, vol. 32, no. 12, pp. 1543-1550, 2016.

[7] C. Tacconi, C. Correale, A. Gandelli et al., "Vascular endothelial growth factor $\mathrm{C}$ disrupts the endothelial lymphatic barrier to promote colorectal cancer invasion," Gastroenterology, vol. 148, no. 7, pp. 1438-1451, 2015.

[8] X. Li, X. Wang, Z. Li, Z. Zhang, and Y. Zhang, "Chemokine receptor 7 targets the vascular endothelial growth factor via the AKT/ERK pathway to regulate angiogenesis in colon cancer," Cancer Medicine, vol. 8, no. 11, pp. 5327-5340, 2019.
[9] V. B. Surya Prasath, D. Vorotnikov, R. Pelapur, S. Jose, G. Seetharaman, and K. Palaniappan, "Multiscale tikhonovtotal variation image restoration using spatially varying edge coherence exponent," IEEE Transactions on Image Processing, vol. 24, no. 12, pp. 5220-5235, 2015.

[10] J. C. Chedjou and K. Kyamakya, "A universal concept based on cellular neural networks for ultrafast and flexible solving of differential equations," IEEE Transactions on Neural Networks and Learning Systems, vol. 26, no. 4, pp. 749-762, 2015.

[11] S. M. Telloni, "Tumor staging and grading: a primer," Methods in Molecular Biology, vol. 1606, pp. 1-17, 2017.

[12] F. S. Hodi, M. Ballinger, B. Lyons et al., "Immune-modified response evaluation criteria in solid tumors (imRECIST): refining guidelines to assess the clinical benefit of cancer immunotherapy," Journal of Clinical Oncology, vol. 36, no. 9, pp. 850-858, 2018.

[13] C. E. L. Klaver, T. M. Kappen, W. A. A. Borstlap, W. A. Bemelman, and P. J. Tanis, "Laparoscopic surgery for T4 colon cancer: a systematic review and meta-analysis," Surgical Endoscopy, vol. 31, no. 12, pp. 4902-4912, 2017.

[14] A. Dehal, A. N. Graff-Baker, B. Vuong et al., "Neoadjuvant chemotherapy improves survival in patients with clinical T4b colon cancer," Journal of Gastrointestinal Surgery, vol. 22, no. 2, pp. 242-249, 2018.

[15] G. Kotronoulas, C. Papadopoulou, K. Burns-Cunningham, M. Simpson, and R. Maguire, "A systematic review of the supportive care needs of people living with and beyond cancer of the colon and/or rectum," European Journal of Oncology Nursing, vol. 29, pp. 60-70, 2017.

[16] L. Bian, D. Wu, Y. Chen et al., "Clinical value of multi-slice spiral CT angiography, colon imaging, and image fusion in the preoperative evaluation of laparoscopic complete mesocolic excision for right colon cancer: a prospective randomized trial," Journal of Gastrointestinal Surgery, vol. 24 , no. 12 , pp. $2822-2828,2020$.

[17] C. Ridereau-Zins, "Imaging in colonic cancer," Diagnostic and Interventional Imaging, vol. 95, no. 5, pp. 475-483, 2014.

[18] M. Svrcek, O. Lascols, R. Cohen et al., "MSI/MMR-deficient tumor diagnosis: which standard for screening and for diagnosis? Diagnostic modalities for the colon and other sites: differences between tumors," Bulletin du Cancer, vol. 106, no. 2, pp. 119-128, 2019.

[19] S. Guo, R. Chen, H. Li, T. Zhang, and Y. Liu, "Identify severity bug report with distribution imbalance by CR-SMOTE and ELM," International Journal of Software Engineering and Knowledge Engineering, vol. 29, no. 2, pp. 139-175, 2019.

[20] X. Zhou, X. Fan, D. Mustafi et al., "Comparison of DCE-MRI of murine model cancers with a low dose and high dose of contrast agent," Physica Medica, vol. 81, pp. 31-39, 2021. 